\title{
Full-Field Terahertz Imaging at Kilohertz Frame Rates Using Atomic Vapor
}

\author{
Lucy A. Downes, ${ }^{1,}$ Andrew R. MacKellar $\odot,{ }^{1}$ Daniel J. Whiting, ${ }^{1}$ Cyril Bourgenot $\odot,{ }^{2}$ \\ Charles S. Adams, ${ }^{1}$ and Kevin J. Weatherill ${ }^{1}$ \\ ${ }^{1}$ Joint Quantum Centre (Durham-Newcastle), Department of Physics, Durham University, \\ South Road, Durham DH1 3LE, United Kingdom \\ ${ }^{2}$ Centre for Advanced Instrumentation, Department of Physics, Durham University, \\ NETPark Research Institute, Joseph Swan Road, Sedgefield TS21 3FB, United Kingdom
}

(Received 28 May 2019; revised manuscript received 15 November 2019; accepted 6 January 2020; published 7 February 2020)

\begin{abstract}
There is much interest in employing terahertz $(\mathrm{THz})$ radiation across a range of imaging applications, but so far, technologies have struggled to achieve the necessary frame rates. Here, we demonstrate a $\mathrm{THz}$ imaging system based upon efficient THz-to-optical conversion in atomic vapor, where full-field images can be collected at ultrahigh speeds using conventional optical camera technology. For a 0.55-THz field, we show an effective $1-\mathrm{cm}^{2}$ sensor with near diffraction-limited spatial resolution and a minimum detectable power of $(190 \pm 30) \mathrm{fW} \mathrm{s}^{-1 / 2}$ per $(40 \times 40) \mu \mathrm{m}^{2}$ pixel capable of video capture at 3000 frames per second. This combination of speed and sensitivity represents a step change in the state of the art of $\mathrm{THz}$ imaging and will likely lead to its uptake in wider industrial settings.
\end{abstract}

DOI: 10.1103/PhysRevX.10.011027

\section{INTRODUCTION}

The THz band is commonly defined as the section of the electromagnetic (EM) spectrum in the $0.1-10-\mathrm{THz}$ range, lying between the far-infrared (IR) and microwave regions [1]. Its ability to penetrate common materials such as paper, cloth, and plastics makes it a promising candidate for use in nondestructive testing $[2,3]$, while the fact that it is nonionizing gives it potential for use in security screening [4] and biomedical [5] settings. Photonic techniques used for visible and infrared light fail at such long wavelengths, while electronic techniques used for microwave and radiofrequency technologies are unsuitable for the high frequencies of the $\mathrm{THz}$ band. Despite the plethora of potential uses, because $\mathrm{THz}$ sources tend to have lower power and $\mathrm{THz}$ detectors have lower sensitivity than devices in other regions of the EM spectrum, there is limited uptake of technologies operating in the $\mathrm{THz}$ range, a phenomenon referred to as the THz gap [6].

For many proposed applications, the ability to collect image data is key. Numerous THz imaging techniques have been developed, each with its own merits and challenges. Single point detectors can be used to collect high-resolution images pixel by pixel [7] but have yet to demonstrate

\footnotetext{
*lucy.downes@durham.ac.uk
}

Published by the American Physical Society under the terms of the Creative Commons Attribution 4.0 International license. Further distribution of this work must maintain attribution to the author(s) and the published article's title, journal citation, and DOI.
Subject Areas: Atomic and Molecular Physics, Optics

real-time capabilities. For example, early techniques employing thermal detectors such as Golay cells and bolometers were limited to response times on the order of $100 \mathrm{~ms}$ per pixel [8]. While some techniques such as self-mixing in quantum cascade lasers (QCLs) can capture pixel data at significantly higher rates [9], they are limited by the speed at which they can raster between pixels [10]. Systems employing digital mirror devices to create coded apertures can image at spatial resolutions below the diffraction limit [11], but the added processing time means that these techniques are not relevant for a real-time system. Fullfield THz imaging techniques, in which a complete image is collected in a single shot, eliminate the need to scan between individual pixels. These schemes typically consist of arrays of small point detectors such as microbolometers [12,13], field-effect transistors (FETs) [14], or carbon nanotubes [15]. Such arrays can operate up to video frame rates of around $60 \mathrm{~Hz}$; however, their sensitivities are limited, and scaling to large pixel numbers can be challenging [16]. More sensitive arrays such as those based on superconductors [17] suffer similar scaling problems and rely on cryogenic cooling to overcome thermal noise, making them cumbersome, expensive, and complex to operate. Another promising approach to full-field $\mathrm{THz}$ imaging is frequency up-conversion, where a nonlinear optical material such as diamond [18] or DAST crystal [19] is used to convert incident $\mathrm{THz}$ photons to more easily detectable wavelengths, allowing images to be collected using standard optical or IR cameras. Despite the high demand, a roomtemperature $\mathrm{THz}$ imaging system combining both high speed and sensitivity has yet to be realized $[1,6,7]$. 
Sensors based on atomic vapor can offer high performance across a range of applications. Because atoms have well-known properties and give reproducible measurements that can easily be related to SI units, they are ideal candidates for use in a new generation of sensors in the growing field of quantum technologies [20]. For example, atom-based sensors have been used for precise measurements of magnetic fields [21,22] and gravity $[23,24]$ and are beginning to be marketed and used in fields as diverse as biomedical sensing [25], geomagnetic [26], and defense applications [27]. A subclass of atom-based sensors includes those based upon Rydberg atoms, atoms that have been excited to a high principal quantum number $n$. It has been shown that Rydberg atoms are excellent sensors for weak electromagnetic fields in the radio-frequency, microwave, and $\mathrm{THz}$ ranges [28-31], as well as for trace gas detection [32]. Recently, the ability of Rydberg atoms to provide IRto-optical conversion has been exploited [33-35], and imaging of high-power pulsed IR [36] and $\mathrm{THz}$ [37] fields has been demonstrated using a Rydberg atom photocathode.

In this work, we demonstrate and characterize a $\mathrm{THz}$ imaging system based on the principal of THz-to-optical conversion in a room-temperature atomic vapor [38]. For a $0.55-\mathrm{THz}$ field, we show an effective $1-\mathrm{cm}^{2}$ sensor with near diffraction-limited spatial resolution and a minimum detectable power of $(190 \pm 30) \mathrm{fW} \mathrm{s}^{-1 / 2} \operatorname{per}(40 \times 40) \mu \mathrm{m}^{2}$ pixel. We show that the system is capable of high-speed imaging by demonstrating video capture at 3000 frames per second, rates that are impossible with other available $\mathrm{THz}$ technologies. Since the speed is ultimately limited by the atomic lifetime of $0.80 \mu \mathrm{s}$, we expect that frame rates of up to $1 \mathrm{MHz}$ would be possible. In Sec. II, we describe the atomic system at work in the conversion process and detail how we make use of this to perform $2 \mathrm{D} \mathrm{THz}$ imaging. In Sec. III, we characterize the spatial and temporal resolution of the system and measure the minimum detectable power. We discuss the limitations of the system presented here and highlight future modifications and improvements in Sec. IV.

\section{EXPERIMENTAL SETUP}

The principle of operation is to use caesium Rydberg atoms to perform THz-to-optical frequency conversion, converting difficult-to-detect $\mathrm{THz}$ radiation into easy-todetect optical photons. The electric dipole transitions between neighboring Rydberg states of alkali atoms lie in the $\mathrm{THz}$ frequency range [39]. These transitions have very large dipole moments, resulting in a high probability of interaction with a resonant $\mathrm{THz}$ field. Rydberg states are relatively long-lived, with natural lifetimes on the order of microseconds, and they decay via spontaneous emission back to the atomic ground state. While this decay process is probabilistic, the complex atomic structure results in certain pathways being more likely than others, leading to reasonable THz-to-optical conversion efficiencies. In this work, we use a $0.55-\mathrm{THz}$ field to couple the $n=14$ and $n^{\prime}=13$ levels in caesium. For each $\mathrm{THz}$ photon absorbed by the system, we have a $52.4 \%$ chance of emitting a signal photon at $535 \mathrm{~nm}$, allowing us to perform THz-to-optical conversion. By creating a 2D sheet of atoms, we use the emitted visible photons to create a full-field image of the incident $\mathrm{THz}$ field in a single exposure. The versatility of this approach means that any optical camera can be employed and can easily be substituted into the system.

We use atomic caesium due to its high vapor pressure at room temperature and easily accessible transition wavelengths. The atomic structure involved in the process is shown in Fig. 1(a). The vapor is prepared in the $14 \mathrm{P}_{3 / 2}$ state using IR lasers (red arrows) in a three-step excitation process [40]. The probe laser ( $5 \mathrm{~mW}$ at $852 \mathrm{~nm})$ excites atoms to the $6 \mathrm{P}_{3 / 2}$ state, and the coupling laser $(20 \mathrm{~mW}$ at $1470 \mathrm{~nm})$ takes atoms from the $6 \mathrm{P}_{3 / 2}$ state to the $7 \mathrm{~S}_{1 / 2}$ state. Both lasers are commercial ECDLs with sub-MHz linewidths and are frequency stabilized to an atomic resonance. The probe laser is stabilized to the $6 \mathrm{~S}_{1 / 2} \mathrm{~F}=4 \rightarrow 6 \mathrm{P}_{3 / 2} \mathrm{~F}^{\prime}=5$ hyperfine transition using polarization spectroscopy [41], while the coupling laser is stabilised to the $6 \mathrm{P}_{3 / 2} \mathrm{~F}^{\prime}=5 \rightarrow$ $7 \mathrm{~S}_{1 / 2} \mathrm{~F}^{\prime \prime}=4$ using excited-state polarization spectroscopy [42]. The Rydberg laser ( $200 \mathrm{~mW}$ at $843 \mathrm{~nm}$ ) is tuned to the $7 \mathrm{~S}_{1 / 2} \rightarrow 14 \mathrm{P}_{3 / 2}$ transition and is not actively frequency stabilized. Once prepared in the $14 \mathrm{P}_{3 / 2}$ state, a resonant $\mathrm{THz}$ field $(17 \mu \mathrm{W}$ at $0.55 \mathrm{THz})$ transfers population into the nearby $13 \mathrm{D}_{5 / 2}$ state, shown by the purple arrow in Fig. 1(a). This narrow-band continuous-wave $\mathrm{THz}$ field is generated using an amplifier multiplier chain (AMC), manufactured by Virginia Diodes Inc., which is seeded by a microwave signal generator.

Both Rydberg states can decay back to the ground state through the emission of visible photons, the wavelength of which depends on the decay pathway taken. We use an Ocean Optics USB spectrometer to measure the spectrum of the emitted fluorescence [Fig. 1(b)], both with and without the $\mathrm{THz}$ field (orange and green lines, respectively). In the absence of the $\mathrm{THz}$ field, the $14 \mathrm{P}_{3 / 2}$ state is populated and subsequently decays via multiple pathways with similar probabilities, resulting in visible fluorescence comprising multiple different wavelengths. We refer to this fluorescence as the " $\mathrm{THz}_{\text {off }}$ " signal, represented by the orange lines in Figs. 1(a) and 1(b). In contrast, in the presence of the $\mathrm{THz}$ field, population is transferred into the $13 \mathrm{D}_{5 / 2}$ state, which predominantly decays via the intermediate $6 \mathrm{P}_{3 / 2}$ state, resulting in the emission of green $(535-\mathrm{nm})$ photons. We refer to this as the " $\mathrm{THz}_{\text {on }}$ " signal, which is represented by the green lines in Figs. 1(a) and 1(b). Because the emissions from the $14 \mathrm{P}$ and $13 \mathrm{D}$ states at $535 \mathrm{~nm}$ are very different, we can use a narrow-band filter [shaded region in Fig. 1(b)] to effectively isolate the fluorescence from the $\mathrm{THz}$-coupled $13 \mathrm{D}_{5 / 2}$ state and hence increase the signal-to-noise ratio of the system. Since the atomic transition addressed by the $\mathrm{THz}$ 
(a)
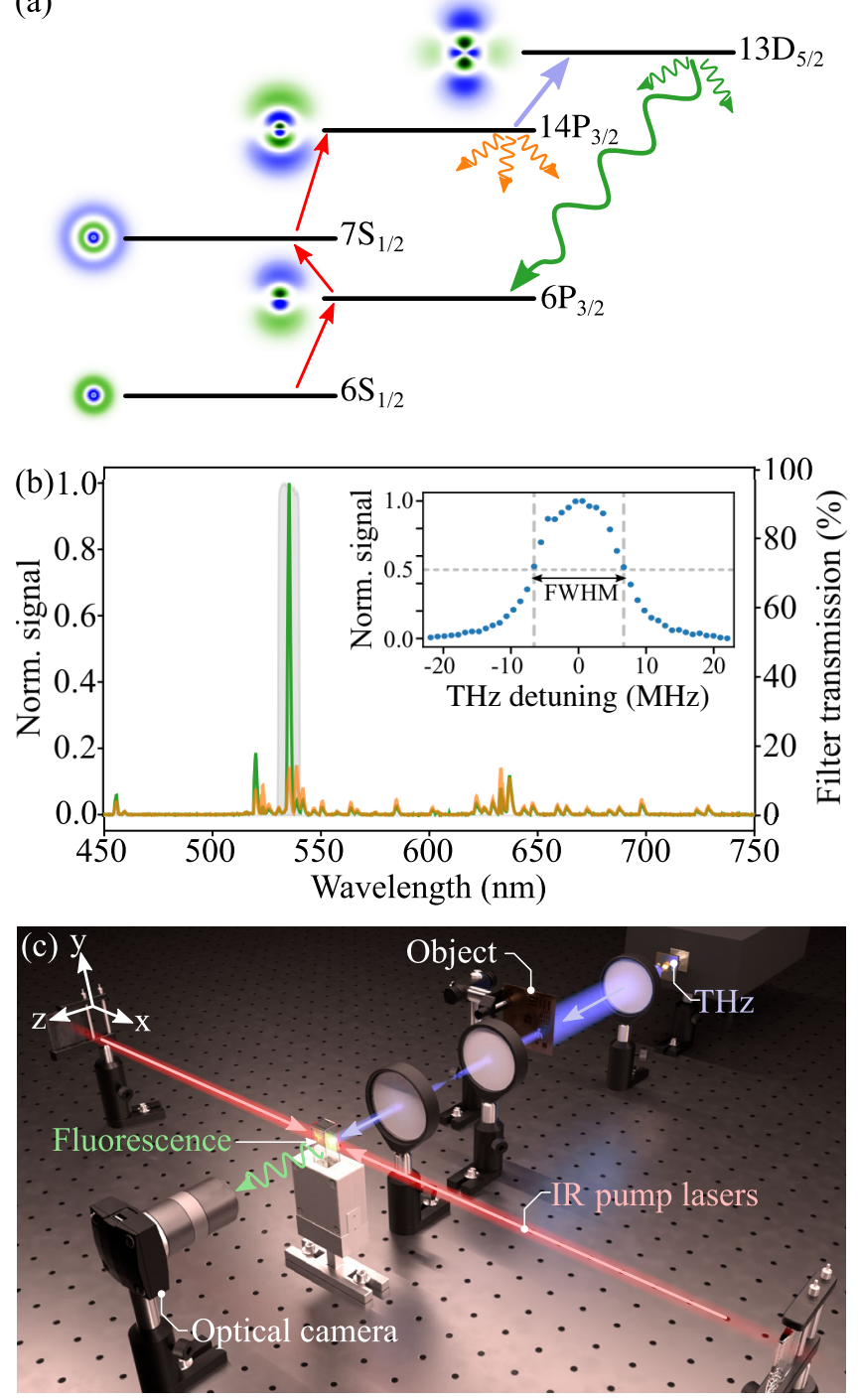

FIG. 1. THz imaging using atomic vapor. (a) The internal energy structure of caesium, highlighting the excitation and principal decay pathways used in this work. The red arrows correspond to laser excitations, the purple arrow to the excitation driven by the $\mathrm{THz}$ field, and the orange and green arrows to optical fluorescence emitted by decay from the $14 \mathrm{P}_{3 / 2}$ and $13 \mathrm{D}_{5 / 2}$ states, respectively. The large green arrow represents the principal decay pathway. (b) The spectral characteristics of the fluorescence from the vapor, both with and without the $\mathrm{THz}$ field (green and orange lines, respectively). The shaded region highlights the optical filter used to isolate the spectral region of interest. Inset: The dependence of the fluorescence signal on $\mathrm{THz}$ detuning, from which we extract a linewidth of $(14.0 \pm 0.4) \mathrm{MHz}$. (c) A diagram of the imaging setup described in this work. Infrared pump lasers form a sheet of excited caesium atoms contained within a quartz cell. The $\mathrm{THz}$ field passes through an object that is imaged onto the atoms using PTFE lenses. Interaction of the $\mathrm{THz}$ field with the excited atoms leads to the emission of green fluorescence, which is captured using an optical camera. field is a narrow-band transition, we expect the system to have a similarly narrow-band response. To determine the linewidth of the response, we record fluorescence spectra at various values of $\mathrm{THz}$ detuning and extract the total signal $\left(\mathrm{THz}_{\mathrm{on}}-\mathrm{THz}_{\text {off }}\right)$ within the filter transmission window [inset of Fig. 1(b)]. From this result, we measure a full width at half maximum (FWHM) of $(14.0 \pm 0.4) \mathrm{MHz}$, as indicated by the grey dashed lines. The structure seen in the data around zero detuning arises from the unresolved hyperfine structure of the Rydberg states.

We create a practical $\mathrm{THz}$ imaging sensor using our atomic THz-to-optical conversion process by forming a 2D sheet of excited atoms onto which a THz-field image can be projected. A schematic of this imaging setup is shown in Fig. 1(c). The caesium vapor is contained within a quartz cell and heated to around $50{ }^{\circ} \mathrm{C}$ to increase the vapor pressure and maximize the resulting fluorescence signal. All three laser beams propagate coaxially and are shaped to form a light sheet of approximately $100 \mu \mathrm{m}$ in width and $10 \mathrm{~mm}$ in height at the position of the vapor cell. The Rydberg laser propagates in the opposite sense to the probe and coupling beams along the $x$ axis. The object to be imaged is illuminated with a continuous-wave $\mathrm{THz}$ field, which propagates in the $z$ direction, perpendicular to the plane of the excited vapor, and is linearly polarized in the vertical $(y)$ direction. Along the axis of this beam, a $1: 1$ transmission imaging system, comprised of polytetrafluoroethylene (PTFE) aspheric lenses, is used to project a $\mathrm{THz}$ image onto the sheet of excited atoms. The fluorescence emitted by atoms in the region of overlap between the lasers and the $\mathrm{THz}$ beam is reimaged onto an optical camera, providing an image of the incident THz field in a single shot. In this work, three different optical cameras were employed. The true-color images were taken using a commercial Nikon D5500 DSLR (camera A) with an exposure time of $0.5 \mathrm{~s}$, an F-stop of f / 1.8, and an ISO of 100. An Andor iXon EMCCD camera (camera B) was used for high-resolution low-noise images, running without water cooling to the EMCCD. An exposure time of $200 \mathrm{~ms}$ was used for this camera, with the EM gain set to minimum. A Photron FASTCAM SA4 (camera C) was used to record the high-speed video at exposure times equal to the reciprocal of the frame rates stated. When using cameras B and C, we use a simple 1:1 optical triplet lens to image the vapor and spectral filters to remove unwanted IR scatter and background fluorescence [shaded region in Fig. 1(b)].

\section{CHARACTERIZATION}

To ascertain the spatial resolution of the system, we image a metallic "test card" comprising apertures of varying sizes and shapes [see Fig. 2(a)]. Images of a 0.50 -mm-diameter pinhole and " $\Psi$ "-shaped aperture are shown both as true-color unprocessed photographs taken with camera $\mathrm{A}$ and as false-color filtered images taken with camera B. By considering the image of the $0.50-\mathrm{mm}$ - 

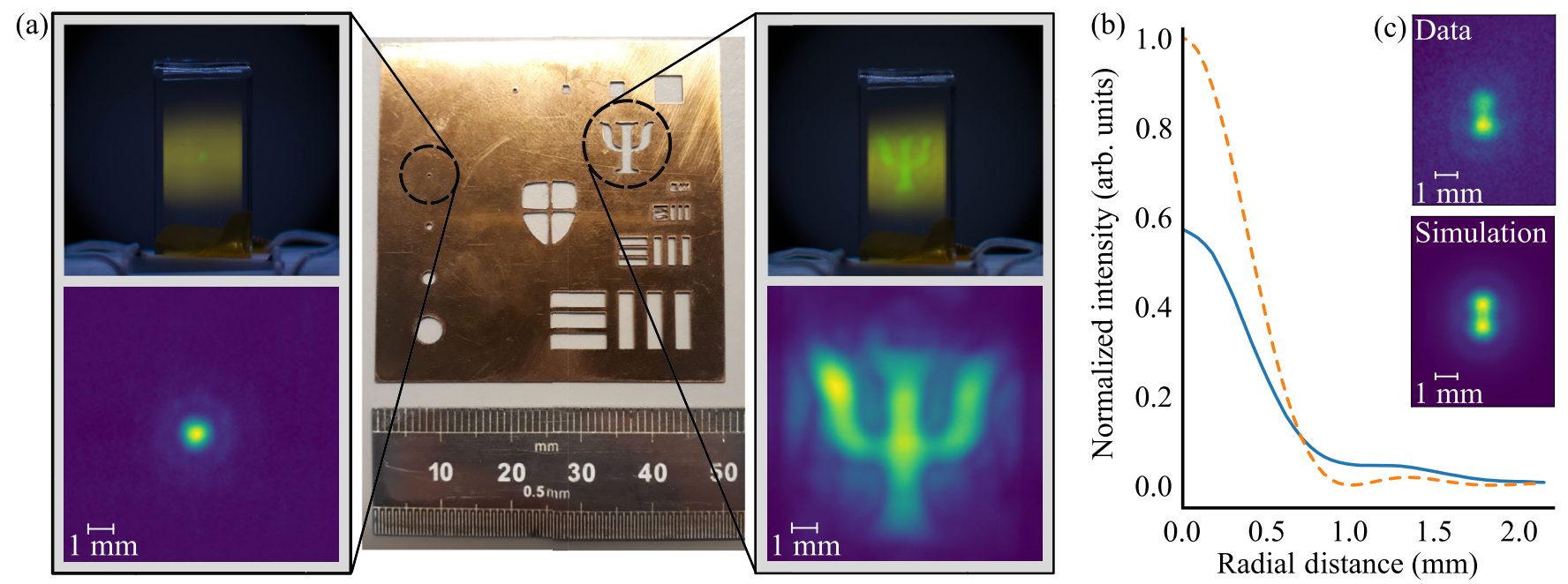

FIG. 2. Demonstration of spatial resolution. (a) A metal mask (center) placed in the object plane of the system. To the left and right are true-color images taken with camera A (above) and false-color images taken with camera B (below) for a 0.50-mm-diameter pinhole (left) and a " $\Psi$ "-shaped aperture (right). (b) Radially averaged intensity profiles of the measured PSF (solid blue line) and the simulated ideal PSF (dashed orange line). Both PSFs were scaled such that the total power in each image was equal. The ratio of peak heights gives a Strehl ratio of 0.57 . (c) Real (top panel) and simulated (bottom panel) images of two 0.50-mm-diameter pinholes separated by $1.00 \mathrm{~mm}$.

diameter pinhole to be the point spread function (PSF) of the system, we are able to compare the performance to that of an ideal imaging system. Since the diameter of the pinhole is subwavelength, we assume that it can be treated as a point source and hence that the ideal intensity distribution will be given by an Airy pattern [43]. Based on the characteristics of the $\mathrm{THz}$ lenses, we calculate the f-number of the system to be 1.5. We normalize both the real and ideal unaberrated PSF such that the total power in each image is equal, and then perform a radial average
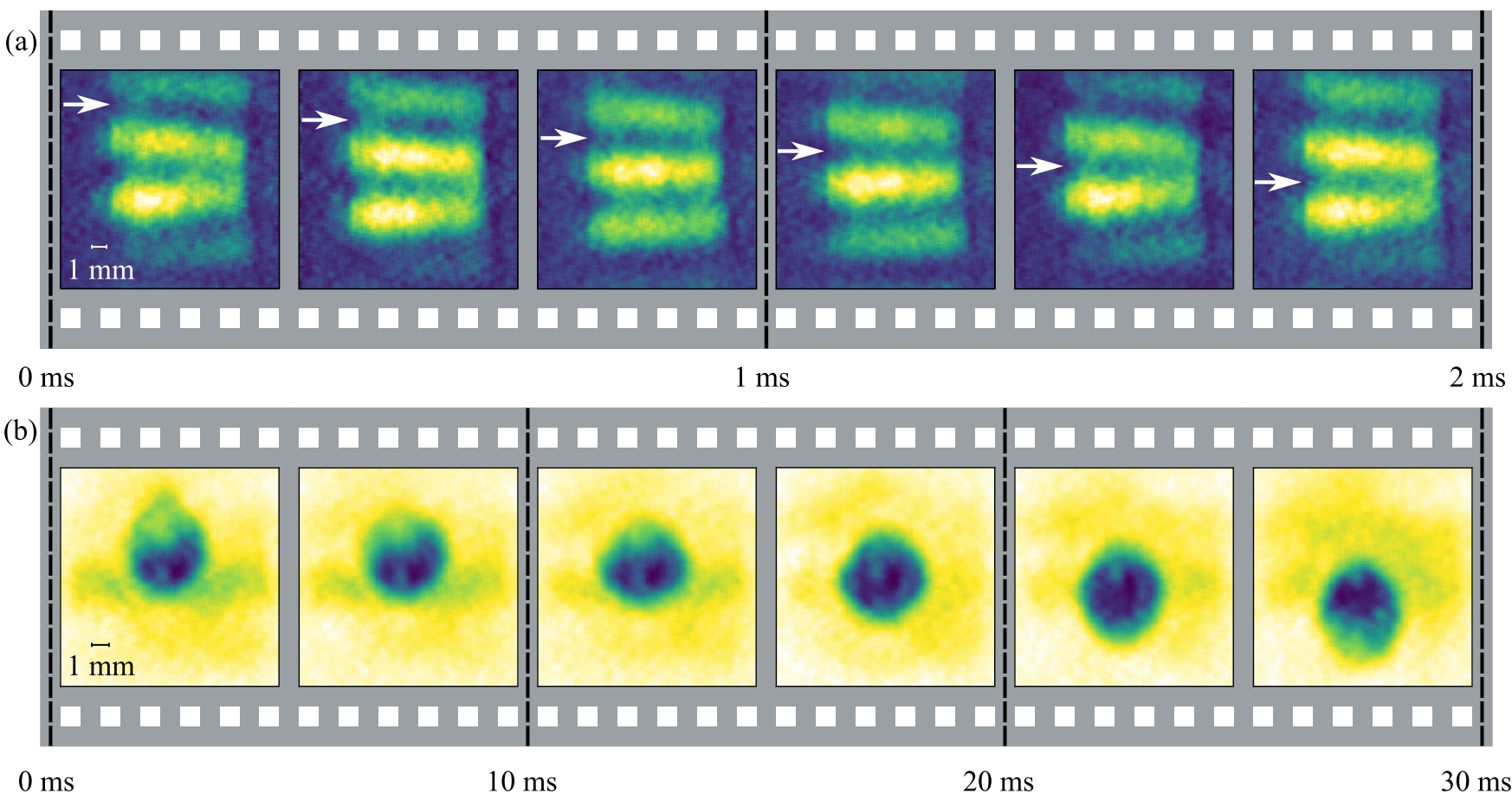

FIG. 3. Ultrahigh-speed THz video. (a) Subsequent frames from a THz video of an optical chopper wheel rotating at $700 \mathrm{rpm}$, imaged at a frame rate of $3 \mathrm{kHz}$. The white arrow is added to highlight the movement of one spoke of the wheel between frames. (b) A significantly slower frame rate of $500 \mathrm{~Hz}$, is sufficient to capture the dynamics of a water droplet in free fall shortly after being released from a buret, every fourth frame of which is shown here. Full video files are available online [44]. 
about the point of peak intensity, the results of which are plotted in Fig. 2(b). By calculating the ratio of the maximum peak heights, we extract a Strehl ratio for our system of 0.57 , indicative of a moderately aberrant system. We posit that these aberrations arise from the simple design of the $\mathrm{THz}$ lenses used. By considering the Rayleigh criterion, we expect that our system will be able to resolve two point sources separated by $1.00 \mathrm{~mm}$. We demonstrate this ability by imaging two 0.50 -mm-diameter pinholes separated by $1.00 \mathrm{~mm}$ [Fig. 2(c)]. The resulting image (top panel) closely resembles the simulated image (bottom panel) in which the two distinct apertures are clearly resolved, providing an upper bound of $1.00 \mathrm{~mm}$ on the system's spatial resolution. We conclude that the system achieves near-diffraction-limited resolution and is currently limited by the simple design of the THz lens system used.

To illustrate the high-speed capabilities of this technique, we demonstrate $\mathrm{THz}$ imaging of dynamical processes at frame rates up to $3 \mathrm{kHz}, 2$ orders of magnitude faster than the current state of the art [19]. Here, we use camera $C$ to capture videos of a water droplet in free fall at a frame rate of $500 \mathrm{~Hz}$ and a rotating optical chopper wheel at $3 \mathrm{kHz}$. Frames from each video are presented in Fig. 3 (full video files are available in the Supplemental Material [44]). Although the frames in Fig. 3(b) were taken at a frame rate of $500 \mathrm{~Hz}$, only every fourth frame is shown to illustrate the changing shape of the water droplet in free fall captured in the video. To improve image clarity, we perform postprocessing on the captured frames, further details of which can be found in the Supplemental Material [44].

We characterize the detector sensitivity by measuring the minimum detectable power (MDP): the minimum $\mathrm{THz}$ power at which the resulting optical signal is reliably detectable above the noise. We use camera B to record a series of images, both with and without the incident 0.55 $\mathrm{THz}$ field, for varying $\mathrm{THz}$ powers. We define the signal as the pixel value resulting from fluorescence in the presence of the $\mathrm{THz}$ field minus that from background fluorescence in the absence of the $\mathrm{THz}$ field, $\mathrm{THz}_{\text {on }}-\mathrm{THz}_{\text {off }}$. For a typical $(40 \times 40) \mu \mathrm{m}^{2}$ pixel close to the center of the image, this background-subtracted signal and its uncertainty are plotted against the incident $\mathrm{THz}$ power in Fig. 4 [45]. We find that the system responds linearly for $\mathrm{THz}$ powers up to around $20 \mathrm{pW}$ per $(40 \times 40) \mu \mathrm{m}^{2}$ pixel, as indicated by the green line. Above this value, the system experiences saturation, and we observe a smaller increase in signal for a given increase in $\mathrm{THz}$ power, which is seen as the deviation from the linear trend at high $\mathrm{THz}$ powers in Fig. 4(a). We determine the MDP by considering the signal in the region of lowest incident $\mathrm{THz}$ power, shown in Fig. 4(b). At exposure times of over $0.5 \mathrm{~s}$, the fluorescence signal saturates the camera, so to obtain an integration time of $1 \mathrm{~s}$, we average over 5 frames, each with an exposure of $200 \mathrm{~ms}$. At this exposure time, we are working

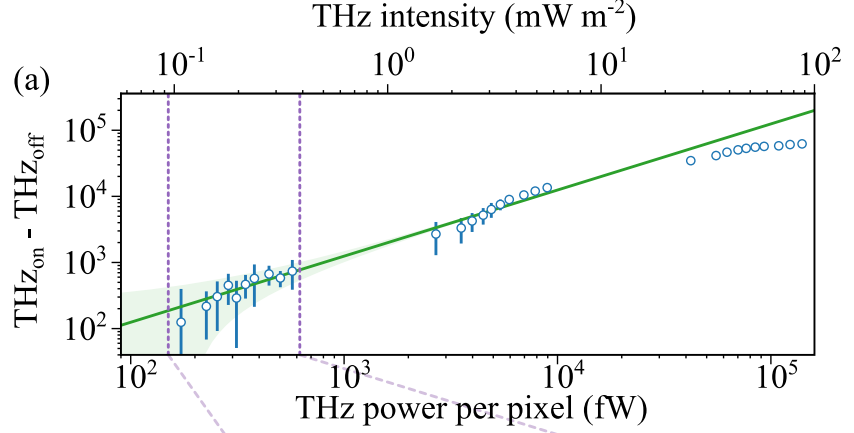

$\mathrm{THz}$ intensity $\left(\mathrm{mW} \mathrm{m}^{-2}\right)$
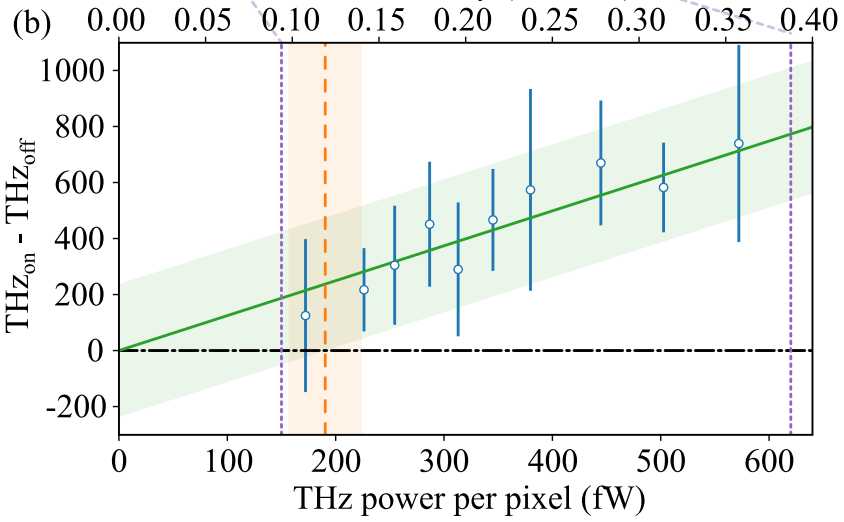

FIG. 4. Minimum detectable power. (a) The fluorescence signal $\mathrm{THz}_{\mathrm{on}}-\mathrm{THz}_{\text {off }}$ (open blue circles) as a function of incident $\mathrm{THz}$ power for a total integration time of 1 second. The linear trendline is extrapolated from that in panel (b) to highlight the saturation point at $\mathrm{THz}$ powers above $20 \mathrm{pW}$ per $(40 \times 40) \mu \mathrm{m}^{2}$ pixel. The vertical dashed lines highlight the range of the data used in panel (b). (b) Plotting the measured fluorescence signal (open blue circles), we can map the linear response of the system (solid green line) plus its associated uncertainty (green shaded region) to the point at which the fluorescence signal is no longer reliably detectable (dashed orange line), at $(190 \pm 30) \mathrm{fW}$ per $(40 \times 40) \mu \mathrm{m}^{2}$ pixel for a 1 -s integration time.

within the shot-noise-limited regime of the camera, where the recorded pixel value scales linearly with exposure time, and the uncertainty is proportional to the square root of this pixel value. In this way, the MDP of our system scales inversely with the square root of the total integrated exposure time used, resulting in a MDP of (190士 30) $\mathrm{fW} \mathrm{s}^{-1 / 2}$ per $(40 \times 40) \mu \mathrm{m}^{2}$ pixel. Alternatively, this result can be expressed as a minimum detectable intensity of $(0.12 \pm 0.02) \mathrm{mW} \mathrm{m}^{-2} \mathrm{~s}^{-1 / 2}$. This value represents an improvement of over 2 orders of magnitude on other roomtemperature imaging systems [16] and is the reason that such high frame rates are possible.

\section{DISCUSSION}

Despite already demonstrating significant improvements in speed and sensitivity over other THz imaging systems, many relatively simple adjustments could be made to 
improve performance further. For example, image quality could be improved by reducing the interference patterns caused by reflections of the $\mathrm{THz}$ field within the cell by making the cell thinner (less than $200 \mu \mathrm{m}$ ) and adding an antireflection coating. Furthermore, the image resolution could be increased by using a more sophisticated $\mathrm{THz}$ lens system or by imaging using higher $\mathrm{THz}$ frequencies by the choice of an appropriate atomic transition from the wide range available [38]. The $\mathrm{THz}$ sensor area could be extended by using a larger vapor cell to enable formation of a larger sheet of excited atoms; however, increased laser powers would be required to maintain laser beam intensity over a larger area. We note that the response time of this system is ultimately limited by the lifetime of the atomic state used, which here is $0.80 \mu \mathrm{s}$ [46]. Our full-field approach enables the system to be used for capturing high-speed $\mathrm{THz}$ video, potentially up to $\mathrm{MHz}$ frame rates with a suitable optical camera. As camera $\mathrm{C}$ is not designed for low-light applications, we are limited in this work to frame rates less than $4 \mathrm{kHz}$; a more sensitive camera would increase the maximum frame rate of the system. We estimate that only $0.4 \%$ of the emitted fluorescence is collected by the current camera lens; therefore, a larger optical lens allowing for greater collection efficiency would also allow for increased frame rates. The wide range of $\mathrm{THz}$ frequency transitions within caesium and other alkali metal atoms means that this system could be extended to operate at multiple $\mathrm{THz}$ frequencies, allowing for "multicolored" $\mathrm{THz}$ imaging. This case could enable spectroscopic information to be collected about a sample alongside transmission images. This high-speed THz imaging system adds to the growing field of quantum technologies. Much of the underlying laser and vapor cell technology has been miniaturized, allowing for the development of portable and affordable quantum devices. Since our THz imaging system uses the same component parts and underlying techniques as other atom-based devices, it will benefit from the drive to bring these technologies to market.

While we highlight simple modifications that could be made to improve the performance of this system, the figures of merit reported here have enabled $\mathrm{THz}$ imaging of dynamical processes at rates not previously possible by any other method. We predict that the versatility and sensitivity of this atom-based $\mathrm{THz}$ imaging technique will produce a disruptive impact on fields as diverse as biological imaging and production-line quality control $[2,3,6]$.

All data and codes are available online at Ref. [47].

\section{ACKNOWLEDGMENTS}

We thank Del Atkinson, Paul Dean, Ifan Hughes, Matt Jones, Marco Peccianti, and Chris Wade for stimulating discussions. We thank Mike Tarbutt, Ray Sharples, Andrew Gallant, and Claudio Balocco for the loan of equipment. This work is supported by UK EPSRC Grants
No. EP/S015973/1, No. EP/R002061/1, No. EP/M014398/ 1, No. EU.H2020.macQsimal ID:820393, and MSquared lasers.

[1] S.S. Dhillon et al., The 2017 Terahertz Science and Technology Roadmap, J. Phys. D 50, 043001 (2017).

[2] C. Jansen, S. Wietzke, O. Peters, M. Scheller, N. Vieweg, M. Salhi, N. Krumbholz, C. Jördens, T. Hochrein, and M. Koch, Terahertz Imaging: Applications and Perspectives, Appl. Opt. 49, E48 (2010).

[3] P. Jepsen, D. Cooke, and M. Koch, Terahertz Spectroscopy and Imaging: Modern Techniques and Applications, Laser Photonics Rev. 5, 124 (2011)

[4] J. F. Federici, B. Schulkin, F. Huang, D. Gary, R. Barat, F. Oliveira, and D. Zimdars, $\mathrm{THz}$ Imaging and Sensing for Security Applications-Explosives, Weapons and Drugs, Semicond. Sci. Technol. 20, S266 (2005).

[5] R. M. Woodward, V. P. Wallace, R. J. Pye, B. E. Cole, D. D. Arnone, E. H. Linfield, and M. Pepper, Terahertz Pulse Imaging of ex vivo Basal Cell Carcinoma, J. Investigat. Derm. 120, 72 (2003).

[6] D. M. Mittleman, Twenty Years of Terahertz Imaging, Invited, Opt. Express 26, 9417 (2018).

[7] S.-L. Chen, Y.-C. Chang, C. Zhang, J. G. Ok, T. Ling, M. T. Mihnev, T. B. Norris, and L. J. Guo, Efficient Real-Time Detection of Terahertz. Pulse Radiation Based on Photoacoustic Conversion by Carbon Nanotube Nanocomposite, Nat. Photonics 8, 537 (2014).

[8] P. Dean et al., Terahertz Imaging Using Quantum Cascade Lasers-A Review of Systems and Applications, J. Phys. D 47, 374008 (2014).

[9] P. Dean, M. U. Shaukat, S. P. Khanna, S. Chakraborty, M. Lachab, A. Burnett, G. Davies, and E. H. Linfield, Absorption-Sensitive Diffuse Reflection Imaging of Concealed Powders Using a Terahertz Quantum Cascade Laser, Opt. Express 16, 5997 (2008).

[10] N. Rothbart, H. Richter, M. Wienold, L. Schrottke, H. T. Grahn, and H.-W. Hubers, Fast 2-D and 3-D Terahertz. Imaging with a Quantum-Cascade Laser and a Scanning Mirror, IEEE Trans. Terahertz Sci. Technol. 3, 617 (2013).

[11] R. I. Stantchev, B. Sun, S. M. Hornett, P. A. Hobson, G. M. Gibson, M. J. Padgett, and E. Hendry, Noninvasive, NearField Terahertz Imaging of Hidden Objects Using a SinglePixel Detector, Sci. Adv. 2 (2016).

[12] A. W. Lee and Q. Hu, Real-Time, Continuous-Wave Terahertz Imaging by Use of a Microbolometer Focal-Plane Array, Opt. Lett. 30, 2563 (2005).

[13] A. W. M. Lee, B.S. Williams, S. Kumar, Q. Hu, and J. L. Reno, Real-Time Imaging Using a 4.3-THz Quantum Cascade Laser and a $320 \times 240$ Microbolometer FocalPlane Array, IEEE Photonics Technol. Lett. 18, 1415 (2006).

[14] H. Qin, J. Sun, S. Liang, X. Li, X. Yang, Z. He, C. Yu, and Z. Feng, Room-Temperature, Low-Impedance and HighSensitivity Terahertz. Direct Detector Based on Bilayer Graphene Field-Effect Transistor, Carbon 116, 760 (2017). 
[15] D. Suzuki, S. Oda, and Y. Kawano, A Flexible and Wearable Terahertz Scanner, Nat. Photonics 10, 809 (2016).

[16] F. Simoens and J. Meilhan, Terahertz Real-Time Imaging Uncooled Array Based on Antenna- and Cavity-Coupled Bolometers, Phil. Trans. R. Soc. A 372, 20130111 (2014).

[17] S. Ariyoshi, C. Otani, A. Dobroiu, H. Matsuo, H. Sato, T. Taino, K. Kawase, and H. M. Shimizu, Superconducting Detector Array for Terahertz Imaging Applications, Jpn. J. Appl. Phys. 45, 37 (2006).

[18] M. Clerici, D. Faccio, L. Caspani, M. Peccianti, E. Rubino, L. Razzari, F. Légaré, T. Ozaki, and R. Morandotti, CCDBased Imaging and 3D Space-Time Mapping of Terahertz Fields via Kerr Frequency Conversion, Opt. Lett. 38, 1899 (2013).

[19] S. Fan, F. Qi, T. Notake, K. Nawata, Y. Takida, T. Matsukawa, and H. Minamide, Diffraction-Limited Real-Time Terahertz Imaging by Optical Frequency Up-Conversion in a DAST Crystal, Opt. Express 23, 7611 (2015).

[20] W. P. Schleich et al., Quantum Technology: From Research to Application, Appl. Phys. B 122, 130 (2016).

[21] D. Budker and M. Romalis, Optical Magnetometry, Nat. Phys. 3, 227 (2007).

[22] E. K. Dietsche, A. Larrouy, S. Haroche, J. M. Raimond, M. Brune, and S. Gleyzes, High-Sensitivity Magnetometry with a Single Atom in a Superposition of Two Circular Rydberg States, Nat. Phys. 15, 326 (2019).

[23] Z.-K. Hu, B.-L. Sun, X.-C. Duan, M.-K. Zhou, L.-L. Chen, S. Zhan, Q.-Z. Zhang, and J. Luo, Demonstration of an Ultrahigh-Sensitivity Atom-Interferometry Absolute Gravimeter, Phys. Rev. A 88, 043610 (2013).

[24] M. Kritsotakis, S. S. Szigeti, J. A. Dunningham, and S. A. Haine, Optimal Matter-Wave Gravimetry, Phys. Rev. A 98, 023629 (2018).

[25] V. K. Shah and R. T. Wakai, A Compact, High Performance Atomic Magnetometer for Biomedical Applications, Phys. Med. Biol. 58, 8153 (2013).

[26] H. B. Dang, A. C. Maloof, and M. V. Romalis, Ultrahigh Sensitivity Magnetic Field and Magnetization Measurements with an Atomic Magnetometer, Appl. Phys. Lett. 97, 151110 (2010).

[27] R. J. Cooper, D. W. Prescott, P. Matz, K. L. Sauer, N. Dural, M. V. Romalis, E. L. Foley, T. W. Kornack, M. Monti, and J. Okamitsu, Atomic Magnetometer Multisensor Array for $r f$ Interference Mitigation and Unshielded Detection of Nuclear Quadrupole Resonance, Phys. Rev. Applied 6, 064014 (2016).

[28] J. A. Sedlacek, A. Schwettmann, H. Kübler, R. Löw, T. Pfau, and J.P. Shaffer, Microwave Electrometry with Rydberg Atoms in a Vapour Cell Using Bright Atomic Resonances, Nat. Phys. 8, 819 (2012).

[29] H. Q. Fan, S. Kumar, R. Daschner, H. Kübler, and J. P. Shaffer, Subwavelength Microwave Electric-Field Imaging Using Rydberg Atoms Inside Atomic Vapor Cells, Opt. Lett. 39, 3030 (2014).

[30] C. L. Holloway, J. A. Gordon, A. Schwarzkopf, D. A. Anderson, S. A. Miller, N. Thaicharoen, and G. Raithel, Sub-wavelength Imaging and Field Mapping via
Electromagnetically Induced Transparency and AutlerTownes Splitting in Rydberg Atoms, Appl. Phys. Lett. 104, 244102 (2014).

[31] C. G. Wade, M. Marcuzzi, E. Levi, J. M. Kondo, I. Lesanovsky, C.S. Adams, and K. J. Weatherill, A Terahertz-Driven Non-equilibrium Phase Transition in a Room Temperature Atomic Vapour, Nat. Commun. 9, 3567 (2018).

[32] J. Schmidt et al., Proof of Concept for an Optogalvanic Gas Sensor for NO Based on Rydberg Excitations, Appl. Phys. Lett. 113, 011113 (2018).

[33] M. Kiffner, A. Feizpour, K. T. Kaczmarek, D. Jaksch, and J. Nunn, Two-Way Interconversion of Millimeter-Wave and Optical Fields in Rydberg Gases, New J. Phys. 18, 093030 (2016).

[34] T. Vogt, C. Gross, J. Han, S. B. Pal, M. Lam, M. Kiffner, and W. Li, Efficient Microwave-to-Optical Conversion Using Rydberg Atoms, Phys. Rev. A 99, 023832 (2019).

[35] J. P. Covey, A. Sipahigil, and M. Saffman, Microwave-toOptical Conversion via Four-Wave Mixing in a Cold Ytterbium Ensemble, Phys. Rev. A 100, 012307 (2019).

[36] M. Drabbels and L. D. Noordam, Infrared Imaging Camera Based on a Rydberg Atom Photodetector, Appl. Phys. Lett. 74, 1797 (1999).

[37] A. Gürtler, A. S. Meijer, and W. J. van der Zande, Imaging of Terahertz Radiation Using a Rydberg Atom Photocathode, Appl. Phys. Lett. 83, 222 (2003).

[38] C. G. Wade, N. Šibalić, N. R. de Melo, J. M. Kondo, C. S. Adams, and K. J. Weatherill, Real-Time, Near-Field Terahertz Imaging with Atomic Optical Fluorescence, Nat. Photonics 11, 40 (2017).

[39] T. F. Gallagher, Rydberg Atoms, Cambridge Monographs on Atomic, Molecular and Chemical Physics (Cambridge University Press, Cambridge, England, 1994).

[40] C. Carr, M. Tanasittikosol, A. Sargsyan, D. Sarkisyan, C. S. Adams, and K.J. Weatherill, Three-Photon Electromagnetically Induced Transparency Using Rydberg States, Opt. Lett. 37, 3858 (2012).

[41] M. L. Harris, C. S. Adams, S. L. Cornish, I. C. McLeod, E. Tarleton, and I. G. Hughes, Polarization Spectroscopy in Rubidium and Cesium, Phys. Rev. A 73, 062509 (2006).

[42] C. Carr, C. S. Adams, and K. J. Weatherill, Polarization Spectroscopy of an Excited State Transition, Opt. Lett. 37, 118 (2012).

[43] C. S. Adams and I. G. Hughes, Optics f2f, from Fourier to Fresnel (Oxford University, New York, 2019).

[44] See Supplemental Material at http://link.aps.org/ supplemental/10.1103/PhysRevX.10.011027 for details of the image processing, minimum detectable power calculation and full video files from which the frames in Fig. 3 are taken.

[45] I. G. Hughes and T. P. A. Hase, Measurements and their Uncertainties (Oxford University, New York, 2010).

[46] N. Šibalić, J. Pritchard, C. Adams, and K. Weatherill, ARC: An Open-Source Library for Calculating Properties of Alkali Rydberg Atoms, Comput. Phys. Commun. 220, 319 (2017).

[47] https://doi.org/10.15128/r16w924b81j 